\title{
Seguimiento neumológico de los niños con displasia broncopulmonar al alta de la Unidad de Cuidado Intensivo Neonatal \\ Parte 2: Administración de oxígeno, tratamiento farmacológico y seguimiento
}

Follow-up of infants with bronchopulmonary dysplasia after

NICU discharge. Part II: Oxygen administration, farmacological treatment and follow-up

http:/ /dx.doi.org/10.5546/aap.2013.252

Coordinadores: Dra. Verónica Giubergia, Fernando Rentería.

Colaboradores: Dra. Gabriela Bauer, Dra. Hebe González Pena, Dr. Fernando Vila, Dra. Verónica Giubergia, Dra. Alicia Michelini, Dra. Verónica Aguerre, Dr. Conrado Llapur, Dra. Diana Fariña, Dra Dora Haag y Dra. Virginia D’Alessandro

OXIGENOTERAPIA CRÓNICA EN EL PACIENTE CON DISPLASIA BRONCOPULMONAR ESTABLECIDA

Valores de corte para indicar oxigenoterapia crónica

Esta guía acuerda que los niños con DBP deben mantener un nivel de $\mathrm{SaO}_{2} \geq 92 \%$ con el objetivo de evitar la hipertensión pulmonar, favorecer el crecimiento y mejorar la regulación del sueño. ${ }^{6,32-35}$

Los valores propuestos están referidos al lactante que ya ha pasado la etapa neonatal con diagnóstico establecido de DBP, cuando se encuentra en un período estable y de transición desde la internación hacia su domicilio. Se requiere exactitud en las valoraciones para adecuar las necesidades de oxígeno en relación con las demandas tisulares, evitando riesgos por la falta o el exceso de medicación. ${ }^{6,32}$

En los últimos años ha surgido preocupación respecto del punto de corte superior de $\mathrm{SaO}_{2}$ en el niño con DBP establecida. En un estudio multicéntrico evaluaron los valores de $\mathrm{SaO}_{2}$ con relación a la retinopatía del prematuro. ${ }^{36}$ En un grupo los niveles fueron igual o menores de $94 \%$ y en otro grupo entre $95 \%-99 \%$. No se encontraron diferencias en la mortalidad, gravedad de la retinopatía, crecimiento y neurodesarrollo entre los grupos. No obstante, los niños del grupo con $\mathrm{SaO}_{2}>95 \%$ que presentaban DBP, tuvieron mayor frecuencia de trastornos respiratorios.

En otro estudio, los neonatos menores de 30 semanas con DBP que tenían $\mathrm{SaO}_{2}>95 \%$ presentaron mayor estadía hospitalaria y alta con $\mathrm{O}_{2}$ suplementario. ${ }^{37}$

Por lo tanto, mantener valores de $\mathrm{SaO}_{2}$ superiores a $95 \%$ en niños con DBP no produce beneficio alguno y debe evitarse.

Los lactantes sanos de 2 a 6 meses de vida tienen un rango normal de $\mathrm{SaO}_{2}$ entre $97 \%$ y $100 \%$; muchos lactantes con DBP permanecerán con rangos de saturación entre 92\% y $96 \%$. Si esta situación se acompaña de un crecimiento adecuado, sin signos de hipertensión pulmonar, ese rango es aceptable. Durante el primer año de vida los niños con DBP irán normalizando la saturación al respirar aire ambiente.
Recibido: 25-10-2012 Aceptado: 31-1-2013
Conflicto de intereses: Ninguno que declarar. 


\section{Criterios de indicación de oxígeno suplementario}

- Criterio estándar para la población de niños con DPB en general: pacientes cuya $\mathrm{SaO}_{2}$ respirando aire ambiente sea $<92 \%$.

- Criterios clínicos de evaluación individual: considerar la oxigenoterapia en los pacientes cuya $\mathrm{SaO}_{2}$ respirando aire ambiente se encuentre entre $92 \%$ y $96 \%$ y presenten alguna de las siguientes situaciones:

a. Signos de compromiso pulmonar crónico y retraso del crecimiento recibiendo el aporte nutricional requerido. Se debe evaluar la eficacia de la oxigenoterapia para favorecer el crecimiento antes de indicar su uso crónico.

b. Diagnóstico de hipertensión pulmonar. Debe plantearse una evaluación conjunta con el cardiólogo de cabecera.

\section{Medición}

La oximetría de pulso es el método de elección. Presenta una adecuada correlación con la $\mathrm{PO}_{2}$ arterial. ${ }^{38}$

Los períodos de hipoxia aguda, intermitentes o prolongados causan hipertensión pulmonar o falla del crecimiento en los niños con DBP. También se han publicado muertes súbitas atribuibles a eventos de hipoxemia no registrados. Por lo expuesto, se debe evaluar la ocurrencia de hipoxemia durante períodos prolongados de sueño, vigilia, alimentación y llanto. Ante una infección respiratoria aguda o algún procedimiento bajo anestesia, puede aumentar el requerimiento de $\mathrm{O}_{2}$ o requerirlo si no existía antes. En estas circunstancias, debe garantizarse el monitoreo continuo de la $\mathrm{SaO}_{2}$. En los niños con enfermedad pulmonar crónica muy grave o en casos de empeoramiento agudo, podría ser necesaria una gasometría arterial para evaluar la ventilación. ${ }^{6,39}$

\section{Suspensión}

Si el niño evoluciona con crecimiento normal y no presenta signos de hipertensión pulmonar (por ecocardiagrama) durante el último mes de seguimiento, se comienza durante los controles clínicos ambulatorios, con pruebas de $\mathrm{SaO}_{2}$ en alimentación, llanto, vigilia y aire ambiente. Si los valores son $\geq 92 \%$ se comienza a suspender el $\mathrm{O}_{2}$. Esto debe hacerse de manera gradual, incrementando progresivamente las horas sin oxígeno durante la vigilia. ${ }^{6,40}$ Antes de suspender el $\mathrm{O}_{2}$ nocturno se debe indicar una oximetría continua durante el sueño nocturno de al menos 6 horas. En la mayoría de los casos esta práctica se realiza con el niño internado. Se considera fallido el intento si la $\mathrm{SaO}_{2}$ promedio es $<92 \%$ $\mathrm{o}$, independientemente de que esta se encuentre dentro de ese valor, si la ganancia de peso se detuvo habiendo manteniendo el mismo aporte nutricional previo a la suspensión. ${ }^{6,40}$

Durante los primeros 3 meses de la suspensión el paciente debe permanecer con el equipo de oxígeno en su domicilio, ya que si presentaran intercurrencias respiratorias podría requerirlo nuevamente. ${ }^{40}$

Las conductas propuestas son protectoras para la evolución de la mayoría de los niños con DBP. En cada caso debe efectuarse una cuidadosa evaluación clínica individual.

\section{TRATAMIENTO FARMACOLÓGICO EN LA DISPLASIA BRONCOPULMONAR ESTABLECIDA}

No hay fármacos específicos para el tratamiento de la DBP. ${ }^{41}$

\section{Cómo evaluar la necesidad de tratamiento farmacológico en el niño con displasia broncopulmonar}

- Evaluar el estado general y la adaptación del niño a la condición que padece.

- Determinar el patrón y la gravedad del compromiso pulmonar (situación basal) para detectar empeoramientos más fácilmente.

- Realizar controles con oxímetro de pulso para valorar el aumento en los requerimientos de $\mathrm{O}_{2}$.

- Controlar estrictamente el crecimiento como parámetro de buena evolución.

- Replantear la utilidad de los tratamientos que el niño viene recibiendo en forma crónica.

- No indicar una medicación como rutina.

\section{Broncodilatadores}

El uso de broncodilatadores agonistas $\beta 2$ (salbutamol) en la DBP ha sido muy debatido. No hay evidencia suficiente para apoyar o refutar su empleo. Están indicados en los pacientes con signos clínicos o funcionales de obstrucción reversible de la vía aérea y para tratar las exacerbaciones. ${ }^{41}$

Algunos pacientes con antecedentes de DBP grave pueden presentar traqueobroncomalacia. En estos niños los agonistas $\beta 2$ podrían causar una respuesta paradójica, por lo que debe evaluarse la respuesta clínica individual antes de prescribirlos. La administración de estos fármacos en 
los niños con DBP, a cualquier edad que se considere, está limitada a los episodios agudos de obstrucción bronquial. Las pruebas de función pulmonar pueden identificar a aquellos con respuesta positiva a esta medicación. ${ }^{41,42}$ No se han demostrado beneficios con el uso crónico de salbutamol o de broncodilatadores de acción prolongada, como el salmeterol o el formoterol. ${ }^{41}$

Los anticolinérgicos (por ejemplo, bromuro de ipratropio) tienen menor efecto broncodilatador que los agonistas $\beta 2$. Estos fármacos no están recomendados para el manejo de la obstrucción bronquial en el niño con DBP. ${ }^{41,42}$

\section{Diuréticos}

No hay evidencia suficiente para apoyar el uso generalizado de diuréticos en el tratamiento de la DBP. ${ }^{41}$ Su utilización es excepcional en los niños con DBP mayores de un año. Exceptuando a los pacientes que presentan un cortocircuito sistémico-pulmonar con aumento del flujo pulmonar que requiera diuréticos, su prescripción debe estar restringida a los cuadros agudos en los pacientes hipoxémicos con signos de edema pulmonar, más frecuentes en el niño prematuro menor de un año. Menos a menudo algunos niños con compromiso pulmonar muy grave y que presentan signos de mala regulación del agua corporal requieren la administración de diuréticos. Es infrecuente que los pacientes sin requerimientos de $\mathrm{O}_{2}$ suplementario necesiten esta medicación.

El más utilizado es la furosemida en dosis de 1-2 mg/ kg/día, por vía oral o intravenosa, según el cuadro clínico del paciente. Otra alternativa es la hidroclorotiazida $2-3 \mathrm{mg} / \mathrm{kg} /$ día por vía oral cada 12 horas. Al lograr la estabilidad clínica se recomienda intentar su uso en días alternos para disminuir las reacciones adversas.

Se debe evitar el uso crónico de diuréticos para evitar sus efectos secundarios. ${ }^{41,43}$

\section{Corticosteroides inhalados}

El uso preventivo de corticosteroides inhalados en los pacientes con DBP es controvertido. Su indicación debe ser muy cuidadosa en aquellos con enfermedad establecida menores de 2 años (especialmente en menores de 6 meses), ya que no se dispone aún de evidencia suficiente que evalúe su efecto, tanto en el crecimiento pulmonar como en el control de la obstrucción bronquial. No se ha demostrado que reduzcan la incidencia de síntomas. ${ }^{41,44-46}$ En los pacientes con DBP que presentan episodios de sibilancias recurrentes no asociados a infecciones virales o antecedentes de asma familiar, o ante la presencia de episodios graves y reiterados, podría considerarse la realización de una prueba terapéutica con corticosteroides inhalados. Estos pacientes deben ser evaluados por un neumólogo.

\section{Antibióticos}

Se recomiendan en las exacerbaciones bacterianas.

\section{Otros fármacos}

Los mucolíticos, antihistamínicos, antitusígenos y antileucotrienos no deberían utilizarse, ya que no se han realizado estudios aleatorizados que indiquen sus ventajas en los niños con DBP.

\section{INMUNOPROFILAXIS EN LOS NIÑOS CON ANTECEDENTES DE DISPLASIA BRONCOPULMONAR}

Inmunoprofilaxis activa: todos los niños prematuros o de bajo peso deben ser inmunizados a los dos meses de edad cronológica, independientemente de la edad gestacional y del peso, según recomiendan la Sociedad Argentina de Pediatría y la Academia Estadounidense de Pediatría. ${ }^{47,48}$ Debe respetarse el calendario oficial vigente en nuestro país.

\section{Vacunas especiales}

La vacuna contra el virus de la gripe A está indicada en los niños mayores de 6 meses en las dosis ya establecidas (primer año dos dosis con intervalo de 1 mes y segundo año una dosis anual), 2 meses antes del pico estacional invernal mientras persista con signos de DBP. También deben vacunarse los convivientes. La vacuna H1N1 quedará sujeta a la recomendación vigente.

Debe indicarse la vacunación contra la tosferina a todos los convivientes de los recién nacidos prematuros menores de $1500 \mathrm{~g}$ hasta los 6 meses de vida, junto con la vacuna antigripal.

\section{Inmunoprofilaxis pasiva con anticuerpo monoclonal antivirus respiratorio sincicial (palivizumab)}

El palivizumab es un anticuerpo monoclonal humanizado que se liga a la proteína $F$, responsable de la fusión del VRS. ${ }^{49}$ Previene el desarrollo de las formas graves.

Existen recomendaciones de uso de palivizumab para prematuros con y sin DBP en nuestro medio (CEFEN). ${ }^{49} \mathrm{~A}$ partir del año 
2011 el Ministerio de Salud de la Nación ha implementado un programa de prevención de infecciones respiratorias agudas para prematuros de alto riesgo que considera la administración de palivizumab a lactantes en riesgo.

Está indicado para los niños menores de 2 años con DBP que han requerido tratamiento (suplemento de $\mathrm{O}_{2}$, broncodilatadores, diuréticos) en los 6 meses anteriores al inicio de la estación de VRS o que son dados de alta durante su transcurso.

Se recomienda su uso desde abril hasta agosto, una dosis mensual de $15 \mathrm{mg} / \mathrm{kg}$ por vía intramuscular (total 5 dosis). ${ }^{49}$

No tiene utilidad en la infección establecida por VRS ni para evitar la diseminación.

\section{EVOLUCIÓN RESPIRATORIA DE LOS NIÑOS CON DISPLASIA BRONCOPULMONAR}

Los lactantes con DBP presentan distintos grados de gravedad de la enfermedad al ser dados de alta. Es una enfermedad respiratoria crónica de buena evolución para la mayoría de los pacientes, ya que el pulmón tiene un alto potencial de crecimiento en esta etapa de la vida. Algunos niños con enfermedad grave pueden tener un gran impacto en su salud en los primeros años de vida y requerir tiempos prolongados de terapia intensiva, reinternaciones, oxigenoterapia; eventualmente, hay riesgo de muerte.

En todos los casos debe promoverse el crecimiento somático del pulmón a través de una nutrición adecuada y del correcto aporte de oxígeno. ${ }^{6}$

\section{ATENCIÓN DE LOS NIÑOS CON ANTECEDENTES DE DISPLASIA BRONCOPULMONAR EN SITUACIONES ESPECIALES}

\section{Infecciones respiratorias agudas en los primeros 2 años de vida}

La infección respiratoria más frecuente en los niños con DBP durante los primeros meses de vida es la bronquiolitis. El agente causal más frecuente es el virus respiratorio sincicial (VRS). La DBP es un factor de riesgo establecido para la aparición de bronquiolitis más graves. ${ }^{6,50}$ Los lactantes con DBP que presentan una bronquiolitis, los menores de 3 meses y los recientemente egresados de la internación tienen mayor riesgo de hospitalización que los prematuros sin DBP y los lactantes nacidos a término y sanos $(13 \%$ contra $8 \%, 0,8 \%$ a $1,3 \%$ respectivamente).
El mayor riesgo de internación se encuentra entre los lactantes nacidos con edades gestacionales muy bajas y aquellos con DBP grave. Antes de la incorporación del palivizumab, hasta el $50 \%$ de los pacientes requerían hospitalizaciones por bronquiolitis. Actualmente, de acuerdo con los datos aportados por el Ministerio de Salud de la Nación, sólo se internaron $4,3 \%$ de niños en $2011 . .^{9,10,41,51}$ No obstante, el manejo de esta patología continúa siendo un desafío en esa población.

En relación con los factores medioambientales, cabe destacar que la asistencia a la guardería, el tabaquismo domiciliario y el hacinamiento se han asociado con mayor riesgo de infección respiratoria aguda en los menores de 2 años. Se debe evitar la asistencia a la guardería e informar a la familia sobre el riesgo de la exposición al humo de tabaco. Se recomienda que los trabajadores sociales del equipo de salud gestionen la mejora de la condición de vivienda en las familias de niños con DBP en situación de vulnerabilidad. ${ }^{49,52}$

El lactante con DBP que presenta una infección respiratoria aguda baja es un paciente de alto riesgo que debe ser controlado en forma frecuente u hospitalizado, según el estado clínico, hasta la desaparición de los síntomas. Para estos niños no es adecuada la modalidad de internación abreviada ("postas de bronquiolitis"). Asimismo, se debe recordar que las escalas de evaluación de gravedad para los lactantes sanos con bronquiolitis no se recomiendan en los pacientes con antecedentes de DBP. ${ }^{6}$

El tratamiento médico de un lactante con DBP con bronquiolitis es de sostén. El principal tratamiento es el oxígeno y, en casos graves, puede requerirse asistencia ventilatoria mecánica. El uso de fármacos, como los broncodilatadores, está destinado a mejorar la mecánica respiratoria. ${ }^{51}$

\section{Sibilancias recurrentes en el niño con antecedentes de displasia broncopulmonar}

Los niños que nacieron prematuramente, con DBP o sin ella, presentan mayor prevalencia de sibilancias recurrentes no asociadas a infecciones respiratorias entre los 8 y 19 años, aunque los síntomas mejoran progresivamente con el tiempo. ${ }^{41}$

Es frecuente que se clasifique erróneamente como asmáticos a los niños con antecedentes de DBP con sibilancias recurrentes y espirometría con evidencia de obstrucción al flujo aéreo. Estos pacientes son tratados con corticosteroides inhalados aun sin evidencia suficiente que lo 
justifique. ${ }^{45,46} \mathrm{Si}$ bien la DBP y el asma comparten algunas características clínicas, el patrón inflamatorio de la vía aérea que presentan es diferente. La inflamación eosinofílica es característica en el niño con asma. En cambio, en la vía aérea del niño con DBP no se ha podido documentar este tipo de patrón inflamatorio. ${ }^{41}$

Los niños con DBP que presentan episodios de sibilancias recurrentes deben ser evaluados por un neumólogo. Es excepcional la indicación de corticosteroides inhalados a los menores de un año, especialmente, a los menores de 6 meses. En los pacientes con DBP que presentan episodios de sibilancias recurrentes no asociados a infecciones virales o antecedentes de asma familiar, o que presenten episodios graves y reiterados podría justificarse una prueba terapéutica con corticosteroides inhalados. Se sugiere iniciar un tratamiento en dosis bajas (budesonida $200 \mu \mathrm{g} /$ día o equivalente) y evaluar la respuesta. Si no se observa respuesta clínica favorable luego de 3 meses, se recomienda suspender el tratamiento, revisar el diagnóstico y descartar comorbilidades.

No hay evidencia aún de que el uso de broncodilatadores agonistas $\beta 2$ de acción prolongada (salmeterol, formoterol) produzca beneficios, por lo que no deberían indicarse en este grupo de pacientes. ${ }^{41}$

\section{CONTROLES AMBULATORIOS DE LOS LACTANTES CON DISPLASIA BRONCOPULMONAR EN EL PRIMER AÑO}

1. Control estricto del crecimiento: en cada visita se debe registrar el crecimiento en talla y peso del paciente como uno de los mejores indicadores de buena evolución.

2. Monitoreo de la saturación de $\mathrm{O}_{2}$ con oxímetro de pulso: se sugiere efectuar el primer control al iniciar el seguimiento a todos los niños con antecedentes de DBP. Debe evaluarse en distintos momentos del día (vigilia, sueño alimentación, llanto). En los pacientes con indicación de oxigenoterapia domiciliaria deben valorarse las variaciones de la $\mathrm{SaO}_{2}$ disminuyendo el flujo de $\mathrm{O}_{2}$ y respirando aire ambiente. Los controles continuarán hasta 2 meses después de retirado el $\mathrm{O}_{2}$. Si el niño no requiere $\mathrm{O}_{2}$ domiciliario y su evolución es favorable luego del primer mes, no es necesario el control regular de $\mathrm{SaO}_{2}$. Está recomendado en los pacientes con antecedentes de DBP que estén cursando una infección respiratoria aguda.

3. Evaluación cardiológica: está sugerida en los niños con oxigenoterapia domiciliaria cada 3 meses en caso de hipertensión pulmonar, cada 6 meses en aquellos sin hipertensión pulmonar y antes de la suspensión del $\mathrm{O}_{2}$ suplementario.

4. Imágenes: con buena evolución clínica se recomienda realizar una radiografía basal durante el primer año y hasta la normalización, o en cualquier momento que se requiera, con protección abdominal. Las radiografías deben quedar en poder de la familia.

5. Gasometría arterial: la presencia de hipercapnia indica un compromiso pulmonar grave. En estos pacientes, luego del egreso de la internación, los controles deben realizarse en forma mensual hasta normalizar la $\mathrm{PCO}_{2} \mathrm{y}$ en cualquier momento si el niño presenta una intercurrencia respiratoria.

6. Pruebas de función pulmonar: no es un estudio de rutina. Se requiere un equipamiento especial, sólo disponible en algunos centros del país.

7. Endoscopia: se sugiere una interconsulta con un endoscopista respiratorio cuando el lactante presenta signos o síntomas de obstrucción de la vía aérea superior.

8. Laboratorio: está propuesto para el control de la anemia, Ca y $\mathrm{P}$, según las recomendaciones para prematuros. Se debe solicitar un ionograma si el paciente recibe diuréticos o presenta edemas.

9. Estudio de sueño: se debe efectuar ante la sospecha de apneas, o hipoventilación central o periférica.

10. Estudios de deglución y seriada esofagogastroduodenal; pHmetría: se plantean frente a la posibilidad de aspiración bronquial por reflujo o por trastornos en la deglución.

\section{CONTROLES AMBULATORIOS DE LOS PREESCOLARES Y ESCOLARES CON ANTECEDENTES DE DISPLASIA BRONCOPULMONAR}

Control del crecimiento: en cada visita se debe registrar el crecimiento en talla y peso del paciente como uno de los mejores indicadores de buena evolución.

Monitoreo de la saturación de $\mathrm{O}_{2}$ con oxímetro de pulso: si el niño no requiere $\mathrm{O}_{2}$ domiciliario y, su evolución es favorable, no es necesario el control de $\mathrm{SaO}_{2}$. Se recomienda en los pacientes con antecedentes de DBP que estén cursando una infección respiratoria aguda.

Pruebas de función pulmonar: en los centros que cuenten con el equipamiento se 
pueden efectuar pruebas de función pulmonar a los preescolares (oscilometría de impulso, espirometría con respuesta broncodilatadora). En los escolares se recomienda realizar al menos una espirometría y repetir según la evolución del niño. La frecuencia de los estudios depende de los resultados y de la gravedad del paciente.

En caso de intolerancia al ejercicio se sugiere efectuar una prueba de broncoprovocación con ejercicio.

Evaluación cardiológica: en los casos en que se resolvió la hipertensión pulmonar, se deben realizar los controles recomendados para los pacientes sin patología cardíaca (examen cardiovascular y ECG en el momento del ingreso escolar y al completar el desarrollo puberal).

En los que persisten con hipertensión pulmonar, se debe consultar con un cardiólogo infantil que definirá de acuerdo con la clase funcional, el grado de hipertensión pulmonar y la repercusión sobre la función cardíaca la modalidad de seguimiento del paciente (ECG y ecocardiograma Doppler color cada 3, 6, 12 meses).

\section{Participaron en la discusión y redacción de esta Guía:}

Comité Nacional de Neumonología: Dra. Virginia Dalessandro Dr. Victor Pawluk, Dra. Beatriz Reches, Dra. Silvia Pereyro, Dra. Cecilia Lepera, Dra. Anahí Krüger, Dra. María Marta Quiñones, Dra. Graciela Luque, Dra. Karina Melillo, Dra. Karina Moncada, Dra. Nora Díaz, Dra. Araceli Luz Benitez, Dra. Verónica Aguerre, Dra. Hilda Giugno, Dra. Patricia Paba, Dra. Analía Stadelman, Dra. Corina Magadan, Dra. Ana Castiñeiras, Dra. Silvina Smith, Dr. Julio Nadeo, Dra. Marcela Fraga, Dr. Pablo Bodas, Dra. Selva Di Coste, Dra. Sandra Baratta, Dra. Ana Balanzat, Dra. Marcela Capellino, Dra. Gisela Martinchuk, Dra. Yanina Loto, Dra. Alicia Michelini, Dr.Fernando Vila, Dra. Verónica Giubergia, Dr. Daniel Álvarez, Dr. Juan Gagneten, Dra. María Eugenia Bonilla, Dr. Diego Dagnino, Dr. Santiago Vidaurreta, Dra. María Elena Andreottola, Dr. Hernán Talamoni, Dr. Ángel Bonina, Dr. Maximiliano Salim, Dr. Fernando Renteria, Dr. Juan Finocchiaro, Dr. Fernando Adot, Dr. Raúl Robles, Dra. Lina Abram, Dra. Sandra Inwentarz, Dra. Mariela Bonini, Dr. Fernando Ferrero, Dra. Norma González, Dr. Claudio Castaños, Dr. Mario Grenoville, Dr. Federico Gini Cambaceres, Dra. Gabriela Surman, Dra. Nadia Tucci, Dra. Teresita Solís, Dra. Judit Pierini, Dr.
Damián Taire, Dra. Sandra Barria, Dr. Néstor Pisapia, Dra. Silvina Prates, Dra. Elena Bonifacich, Dr. Luis Parra, Dr. Gastón Bolognese, Dr. Mauricio Borda, Dra. Silvia Cipriani, Dr. Gonzalo Colombres, Dr. Fernando Meneghetti, Dr. Patricio Pérez, Dra. Catalina Pinchak, Dra. Clarisa Ferreyra, Dra. María Alejandra Zárate, Dra. Liliana Gallardo, Dra. Alejandra Meneguzzi, Dra. Elsa Bisero, Dr. Mauro González Ross, Dra. Daniela Petti, Dra. Patricia Andreossi, Dra. Vivian Leske, Dra. Patricia Meyer, Dra. Gabriela Bauer, Dr. Conrado Llapur, Dra. Hebe González.

\section{Comité Nacional de Estudios Fetoneonatales} (CEFEN): Dra. Daniela Satragno.

Comité Nacional de Cardiología: Dra. Dora Haag.

\section{BIBLIOGRAFÍA}

1. Northway WHJr, Rosan RD, Porter DY. Pulmonary disease following respiratory therapy of hyalinemembrane disease: Bronchopulmonary dysplasia. NEngl JMed 1967;276(7):35768.

2. Bancalari E, Abdenour GE, Feller R, Gannon J. Bronchopulmonary dysplasia: clinical presentation. JPediatr 1979;95(5 Pt 2):819-23.

3. Shennan AT, Dunn MS, Ohlsson A, Lennox K, Hoskins E. Abnormal pulmonary outcomes in premature infants: prediction from oxygen requirement in the neonatal period. Pediatrics 1988;82(4):527-32.

4. Jobe A,BancalariE. Bronchopulmonary Dysplasia-NICHD/ NHLBI/ORD Workshop Summary. Am J Respir Crit Care Med 2001;163(7):1723-9.

5. FehlmannE,Tapia J, FernándezR, Bancalari A, etal. Impacto del síndrome de dificultad respiratoria en recién nacidos de muy bajo peso de nacimiento: estudio multicéntrico sudamericano. Arch Argent Pediatr 2010;108(5):393-400.

6. Desarrollo temprano-Consenso del grupo de seguiSIBEN para el seguimiento del neurodesarrollo en niños prematuros de MBPN. En: Bauer G, Borbonet D, Morgues $\mathrm{M}$, Sola A. Niños prematuros y sus primeros años de vida. Recomendaciones del grupo de seguimiento de recién nacidos de alto riesgo: SeguiSIBEN. Buenos Aires: Edimed; 2011.

7. Walsh MC, Yao Q, Gettner P, Hale E, et al. Impact of a physiologic definition on bronchopulmonary dysplasia rates. Pediatrics 2004;114(5):1305-11.

8. Ehrenkranz RA, Walsh MC, Vohr BR, Jobe AH, et al. Validation of the National Institutes of Health consensus definition of bronchopulmonary dysplasia. Pediatrics 2005;116(6):1353-60.

9. Eichenwald EC, Stark AR. Management and outcomes of very low birth weight. N Engl J Med 2008;358(16):1700-11.

10. Ministerio de Salud de la Nación. Programa de prevención de IRA en prematuros de alto riesgo atendidos en el sector público de salud. 2011.

11. Bancalari A. Actualización en presentación y patogénesis de la displasia broncopulmonar. Rev Chil Pediatr 2009;80 (3):213-24.

12. Biniwale M, Ehrenkranz R. The role of nutrition in the prevention and management of bronchopulmonary dysplasia. Semin Perinatol 2006;30(4):200-8.

13. Chess PR, D'Angio CT, Pryhuber GS, Maniscalco, WM. 
Pathogenesis of bronchopulmonary dysplasia. Semin Perinatol 2006;30(4):171-8.

14. Bancalari E, Claure N, Sosenko IR. Bronchopulmonary dysplasia: changes in pathogenesis, epidemiology and definition. Semin Neonatol 2003;8(1):63-71.

15. LavoieP,PhamCh,JangK.Heritabilityofbronchopulmonary dysplasia, defined according to the consensus statement of the national institutes of health. Pediatrics 2008;122:(3)47985.

16. Guardia C,Moya FR, Maturana, A, etal. Incidence of "New" $\mathrm{BDP}$ among premature infants receiving prophylactic surfactante therapy. PAS 2008. 2-6 May, Honolulu, Hawaii. E-PAS2008:63520.

17. Tiscornia $\mathrm{C}$, Botto $\mathrm{H}$, Rodríguez H. Patología de la vía aérea quirúrgica. En: Martínez Ferro M, Cannizaro C, Rodríguez S, Rabasa C. Neonatología Quirúrgica. Buenos Aires: Grupo Guía; 2004. Págs.267-83.

18. Friedrich L, Stein R, Pitrez P, Corso A, Jones M. Reduced lung function in healthy preterm infants in the first months of life. Am J Respir Crit Care Med 2006;173(4):442-7.

19. Friedrich F, Pitrez P, Stein R, Goldani M, et al. Growth rate of lung function in healthy preterm infants. Am J Respir Crit Care Med 2007;176(12):1269-73.

20. Tepper RS, Morgan WJ, Cota K, Taussig LM. Expiratory flow limitation in infants with bronchopulmonary dysplasia. J Pediatr 1986(6);109:1040-6.

21. Motoyama EK, Fort MD, Klesh KW, Mutich RL, Guthrie RD. Early onset of airway reactivity in premature infants with bronchopulmonary dysplasia. Am Rev Respir Dis 1987;136(1):50-7.

22. BaraldiE, FilipponeM,Trevisanuto D,ZanardoV,Zacchello F. Pulmonary function until two years of life in infants with bronchopulmonary dysplasia. Am J Respir Crit Care Med 1997;155(1):149-55.

23. Balinotti J, Chakr V, Tiller Ch, Kimmel R, et al. Growth of lung parenchyma in infants and toddlers with chronic lung disease of infancy. Am J Respir Crit Care Med 2010;181(10):1093-7.

24. Fawke J, Lum S, Kirkby K, Hennessy E, et al. Lung function and respiratory symptoms at 11 years in children born extremely preterm: the EPICure study. Am J Respir Crit Care Med 2010;182(2):237-45.

25. Lum S, Kirkby J, Welsh L, Marlow N, et al. Nature and severity of lung function abnormalities in extremely preterm children at 11 years of age. Eur Respir 12011;37(5):1199207.

26. Halvorsen T, Skadberg BT, Eide GE, Roksund OD, et al. Pulmonary outcomein adolescents of extreme preterm birth: a regional cohort study. Acta Paediatr 2004;93(10):1294-300.

27. Welsh L, Kirkby J, Lum S, Odendaal D, et al. The EPICure study: maximal exercise and physical activity in school children born extremely preterm. Thorax 2010;65(2):165-72.

28. Barst RJ, Ertel SI, Beghetti M, Ivy DD. Pulmonary arterial hypertension: a comparison between children and adults. Eur Respir J 2011;37(3):665-77.

29. Abman SH, Groothius JR. Pathophysiology and treatment of DBP. Current issues. Pediatr Clin North Am 1994; 41(2):277-315.

30. Khemani E, McElhinnney DB, Rhein L, Andrade O, et al. Pulmonary artery hypertension in formerly premature infants with bronchopulmonary dysplasia: clinical features and outcomes in the surfactant era. Pediatrics 2007;120(6):1260-9.

31. Parker TA, Abman SH. The pulmonary circulation in bronchopulmonary dysplasia. Semin Neonatol 2003;8(1):5161.

32. Tin $\mathrm{W}$, Wiswell T. Drug therapies in bronchopulmonary dysplasia: debunking the myths. Semin Fetal Neonatal Med
2009;14(6):383-90.

33. Groothius JR, Rosenberg AA. Home oxygen promotes weight gain in infants with bronchopulmonary dysplasia. Am J Dis Child 1987;141(9):992-5.

34. Subhedar NV, Shaw NJ. Changes in pulmonary arterial pressure in preterm infants with chronic lung disease. Arch Dis Child Fetal Neonatal Ed 2000;82(3):F243-7.

35. Singer L, Martin RJ, Hawkins SW, Benson-Szekely LJ, et al. Oxigen desaturation complicates feeding in infants with bronchopulmonary dysplasia after discharge. Pediatrics 1992;90(3):380-4.

36. The STOP-ROP Multicenter Study Group. Supplemental therapeutic oxygen for prethreshold retinopathy of prematurity (STOP-ROP), a randomized,controlled trial. I: primary outcomes. Pediatrics 2000;105(2):295-310.

37. Askie LM, Henderson-Smart DJ, Irwig L, Simpson JM. Oxygen-saturation targets and outcomes in extremely preterm infants. N Engl J Med 2003;349(10):959-67.

38. Walsh MC, Noble LM, Carlo WA, Martin RJ. Relationship of pulse oximetry to arterial oxygen tension in infants. Crit Care Med 1987;15(12):1102-5.

39. Suresh V, Shaw B. Carbon dioxide levels do not predict duration of home oxygen requirement: a retrospective study. J Perinat Med 2002;30(4):333-5.

40. ComitédeNeumonología. Sociedad Argentina dePediatría. Oxigenoterapia domiciliaria en pediatría. Arch Argent Pediatr 2005;103(6):535-44.

41. BaraldiE, FilipponeM.Chroniclung disease after premature birth. N Engl J Med 2007;357(19):1946-55.

42. Ng G, da Silva O, Ohlsson A. Bronchodilators for the prevention and treatment of chroniclung disease in preterm infants. Cochrane Database Syst Rev 2012;6:CD003214

43. Brion L, Primhak R, Ambrosio-Perez I. Diuretics acting on the distal renal tubule for preterm infants with (or developing) chronic lung disease. Cochrane Database Syst Rev 2011;9:CD001817.

44. De Benedictis FM, Bush A. Corticosteroids in respiratory diseases in children. Am J Respir Crit Care Med 2012;185(1):12-23.

45. Chan K N, Silverman M. Increased airway responsiveness in children of low birth weight at school age: effect of topical corticosteroids. Arch Dis Child 1993;69(1):120-4.

46. Pelkonen AS, Hakulinen AL, Hallman M, Turpeinen M. Effect of inhaled budesonide therapy on lung function in schoolchildren born preterm. RespirMed 2001;95(7):565-70.

47. Committee on infectious diseases. Recommended childhood and adolescent immunization schedules united states, 2012. Pediatrics 2012;129(2):385-6.

48. Comité Nacional de Infectología. Prioridades para la incorporación de vacunas al calendario nacional. Recomendaciones de la Sociedad Argentina de Pediatría. 2011 [Citado: 1 de febrero de 2013]. Disponible en: http://www.sap.org.ar/docs/publicaciones / ConsensoVacunas2011.pdf

49. Comité de estudios fetoneonatales (CEFEN). Actualización de las recomendaciones sobre el uso del palivizumab. Subcomisiones y Comités. Arch Argent Pediatr 2007;105(1):6770.

50. Giubergia V, Martinchuk G, Moreno N, Colombres, G, et al. Gravedad de la infección por virus sincicial respiratorio en pacientes con factores de riesgo y sin ellos. Arch Arg Pediatr 2004;102 (5): 330-4.

51. Greenough A, Giffin FJ, Yüksel B. Respiratory morbidity in preschool children born prematurely. Relationship to adverse neonatal events. Acta Paediatr 1996;85(7):772-7.

52. Caudri D, Wijga A, Scholtens S, Kerkhof M, et al. Early daycare is associated with an increase in airway symptoms. Am J Respir Crit Care Med 2009;180(6):491-8. 